\title{
Síntese do cerato de bário pelo método de complexação combinando EDTA-citrato e avaliação catalítica na oxidação do monóxido de carbono testada em reator de leito fixo
}

\section{(Synthesis of barium cerate by EDTA-citrate complexing method and catalytic evaluation under carbon monoxide stream tested in fixed-bed reactor)}

\author{
M. F. Lobato ${ }^{1}$, A. G. dos Santos ${ }^{2 *}$, A. B. Vital', C. P. de Souza ${ }^{1}$ \\ ${ }^{I} D E Q / P P G E Q / L M N R C$, Universidade Federal do Rio Grande do Norte, Campus Universitário, Lagoa Nova, \\ Natal, Brasil 59072-970 \\ ${ }^{2}$ Departamento de Agrotecnologia e Ciências Sociais, Universidade Federal Rural do Semi-Árido, \\ Av. Francisco Mota, 572, Campus Mossoró, Mossoró, RN, Brasil 59625-900 \\ wellobato@hotmail.com,*andarair@ufersa.edu.br,angelicavital.eq@gmail.com,carlson@ufrnet.br
}

\begin{abstract}
Resumo
A perovskita de cerato de bário foi sintetizada com base no método de complexação combinando EDTA-citrato e suas propriedades catalíticas testadas na reação de oxidação do monóxido de carbono em um reator de leito fixo variando a vazão de alimentação $\left(50\right.$ e $\left.100 \mathrm{~mL} \cdot \mathrm{min}^{-1}\right)$ e a temperatura $\left(100\right.$ a $\left.550{ }^{\circ} \mathrm{C}\right)$. A fase cristalina de interesse foi obtida com tamanho de cristalito de 133 $\mathrm{nm}$, junto com uma pequena quantidade de $\mathrm{BaCO}_{3}(3 \%)$. Quando sintetizado pelo método de complexação combinando EDTAcitrato, o cerato de bário apresenta um formato esférico irregular com aglomerados de diferentes tamanhos e com a presença de poros de diferentes tamanhos distribuídos aleatoriamente, sendo sua área superficial específica igual a $2,61 \mathrm{~m}^{2} \cdot \mathrm{g}^{-1}$. $\mathrm{Na}$ vazão de $50 \mathrm{~mL} \cdot \mathrm{min}^{-1}$ ocorreu maior conversão quando comparado com a de $100 \mathrm{~mL} \cdot \mathrm{min}^{-1}$, sendo mais evidente entre 300 e $400{ }^{\circ} \mathrm{C}$. Além disso, na vazão de $50 \mathrm{~mL} \cdot \mathrm{min}^{-1}$ ocorreu conversão total de $\mathrm{CO}$ em $\mathrm{CO}_{2}$ a $400{ }^{\circ} \mathrm{C}$, enquanto que em $100 \mathrm{~mL}$.min ${ }^{-1}$ a conversão foi de aproximadamente $60 \%$. Com o uso da perovskita como catalisador o processo de oxidação é completo a $400{ }^{\circ} \mathrm{C}$, enquanto que na reação sem catalisador a conversão completa é alcançada somente a $550{ }^{\circ} \mathrm{C}$. Apesar da sua pequena área superficial, o $\mathrm{BaCeO}_{3} \mathrm{e}$ altamente reativo na oxidação do monóxido de carbono.

Palavras-chave: perovskita, método de complexação combinando EDTA-citrato, propriedades catalíticas, área superficial, vazão de alimentação, monóxido de carbono.
\end{abstract}

\begin{abstract}
The barium cerate perovskite was synthesized using a methodology based on the EDTA-citrate complexing method and its catalytic

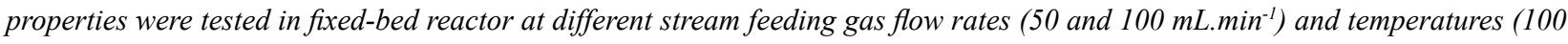
to $550{ }^{\circ} \mathrm{C}$ ). The intended crystalline phase was obtained with crystallite size of $133 \mathrm{~nm}$, together with a small amount of unwanted $\mathrm{BaCO}_{3}$ phase (3\%). When synthesized by the EDTA-citrate complexing method, the barium cerate presents an irregular spherical shape with clusters of different sizes, and the presence of pores of different size distributed randomly; its specific surface area was $2.61 \mathrm{~m}^{2} . \mathrm{g}^{-1}$. At flow rate of $50 \mathrm{~mL} . \mathrm{min}^{-1}$, higher conversion then at $100 \mathrm{~mL}$. $\mathrm{min}^{-1}$ was observed, becoming more apparent between 300 and $400{ }^{\circ} \mathrm{C}$. Moreover, at $50 \mathrm{~mL}$. $\mathrm{min}^{-1}$ flow rate total conversion of $\mathrm{CO}$ to $\mathrm{CO}$ occurred at $400{ }^{\circ} \mathrm{C}$, while the conversion was close to $60 \%$ at $100 \mathrm{~mL} . \mathrm{min}^{-1}$. Using the perovskite as catalyst the oxidation process is completed at $400{ }^{\circ} \mathrm{C}$, while in reaction without catalyst the complete conversion is achieved only at $550{ }^{\circ} \mathrm{C}$. Therefore, the $\mathrm{BaCeO}_{3}$ had strong activity in carbon monoxide oxidation, although its specific area was small.
\end{abstract}

Keywords: perovskite, EDTA-citrate complexing method, catalytic properties, specific area, feed flow, carbon monoxide.

\section{INTRODUÇÃO}

Apesar de apresentar limitações relativas à área superficial, os óxidos do tipo perovskita exibem alto potencial de estabilidade termoquímica a altas temperaturas e, em algumas reações, atividade catalítica [1, 2]. Por exemplo, os materiais à base de niquelatos de lantânio têm demonstrado serem catalisadores promissores em reações de reforma de metano devido às suas propriedades catalíticas e eletrônicas $[3,4]$ ou em reações redox que envolvem óxido de nitrogênio e monóxido de carbono $[5,6]$. Conforme literatura mencionada, tais materiais apresentam atividades catalíticas compatíveis com a apresentada pela platina suportada em alumina $\left(\mathrm{Pt} / \mathrm{Al}_{2} \mathrm{O}_{3}\right)$. Dopada ou não 
com outros elementos, a perovskita de cerato de bário $\left(\mathrm{BaCeO}_{3}\right)$ tem despertado grandes atenções em pesquisas devido às suas possibilidades de aplicações, como em membranas eletrolíticas condutoras de prótons em células combustíveis, por exemplo [7]. Recentemente, Medvedev et al. [8] publicaram uma revisão sobre materiais baseados na perovskita $\mathrm{BaCeO}_{3}$ e suas propriedades. Algumas perovskitas de composições diferentes da binária $\mathrm{BaCeO}_{3}$ foram testadas como catalisador na oxidação do monóxido de carbono, podendo citar: $\mathrm{LaNi}_{0,3} \mathrm{Co}_{0,7} \mathrm{O}_{3-\delta} \mathrm{e} \mathrm{SrCo}_{0,8} \mathrm{Fe}_{0,2} \mathrm{O}_{3-\delta}$ [9], $\quad \mathrm{La}_{0,9} \mathrm{Ba}_{0,1} \mathrm{CoO}_{3} \quad[10], \quad \mathrm{LaCoO}_{3}, \quad \mathrm{LaCo}_{0,8} \mathrm{Cu}_{0,2} \mathrm{O}_{3}$, $\mathrm{La}_{0,8} \mathrm{Sr}_{0,2} \mathrm{Co}_{0,8} \mathrm{Cu}_{0,2} \mathrm{O}_{3}$ e $\mathrm{La}_{0,8} \mathrm{M}_{0,2} \mathrm{FeO}_{3}(\mathrm{M}=\mathrm{Ce}$ e $\mathrm{Sr}$ ) [11]. Tais perovskitas apresentam reatividade e estabilidade com completa conversão de monóxido de carbono em baixas temperaturas de operação $\left(<350{ }^{\circ} \mathrm{C}\right)$ [9-11].

Em termos de aplicações catalíticas do cerato de bário na reação de oxidação do monóxido de carbono na literatura até o presente momento, pode-se verificar seu potencial catalítico, juntamente com a dopagem com 5 e $10 \%$ de paládio $(\mathrm{Pd})$ no sítio $\mathrm{B}$, sob condições similares às da oxidação dos gases de escape de motores movidos a diesel [12]. No referido estudo, os catalisadores foram inicialmente preparados a partir de moagens e aquecimentos controlados a $1000{ }^{\circ} \mathrm{C}$ por $10 \mathrm{~h}$ e testados no formato de pastilhas (16 $\mathrm{mm}$ de diâmetro) num reator de leito fixo. Como resultado, o cerato de bário dopado com $10 \%$ de paládio apresentou maior atividade catalítica $\left(\mathrm{T}_{50}=83^{\circ} \mathrm{C}\right)$, quando comparado com o cerato de bário $\left(\mathrm{T}_{50}=225^{\circ} \mathrm{C}\right)$. Ao diminuir de $10 \%$ para $5 \%$ o teor de paládio no sítio $\mathrm{B}$, também houve uma diminuição na atividade catalítica $\left(\mathrm{T}_{50}=123{ }^{\circ} \mathrm{C}\right)[12]$. O aumento na atividade catalítica está associado ao aumento na dopagem com paládio, o qual atribui maior mobilidade de vacâncias de oxigênio na presença de íons Pd (II) localizados no sítio B da perovskita [13]. Baseado nestas informações e na relevância do método de síntese, o presente trabalho propõe sintetizar o cerato de bário por um método distinto (de complexação combinando EDTA-citrato) da literatura $[12,13]$ e também em formato diferente (pó), e testar seu potencial catalítico na reação de oxidação do monóxido de carbono. É apresentada também como parâmetro inovador uma análise preliminar do efeito da vazão de alimentação gasosa no reator de leito fixo, bem como um comparativo da conversão do monóxido com e sem a presença do catalisador $\left(\mathrm{BaCeO}_{3}\right)$.

\section{EXPERIMENTAL}

Síntese do catalisador $\left(\mathrm{BaCeO}_{3}\right)$ : a perovskita $\mathrm{BaCeO}_{3}$ foi sintetizada com base no método de complexação combinando EDTA-citrato [14, 15]. O método consiste na diluição do EDTA ácido em hidróxido de amônia (1 g: 10 $\mathrm{mL}$ ), sob agitação e aquecimento $\left(40{ }^{\circ} \mathrm{C}\right)$, com posterior introdução dos respectivos metais; e adição do ácido cítrico seguido do aumento da temperatura até $80{ }^{\circ} \mathrm{C}$. A razão molar de EDTA ácido, ácido cítrico e íons metálicos totais de 1,0:1,5:1,0 foi adotada. Posteriormente, fez-se o ajuste do $\mathrm{pH} 11$ com adição de hidróxido de amônia $\left(\mathrm{NH}_{4} \mathrm{OH}\right)$, permanecendo sob agitação e aquecimento até a formação do gel, o qual foi tratado termicamente a $1000{ }^{\circ} \mathrm{C}$ por $5 \mathrm{~h}$.

Caracterizações: a estrutura cristalina da perovskita foi analisada por difração de raios X pelo método do pó, em um difratômetro de raios X (Shimadzu DRX-6000), com radiação $\mathrm{CuK} \alpha$ (comprimento de onda de $1,5406 \AA$ ) e $2 \theta$ variando de 10 a $80^{\circ}$, passo de $0,02^{\circ}, 0,6 \mathrm{~s} /$ passo e análise com o software X'pert HighScore Plus. Para obtenção da melhor concordância possível entre o padrão de difração teórico e o experimental [16], foi feito o refinamento pelo método de Rietveld utilizando o programa Material Analysis Using Diffration (MAUD) v. 2.064, também usado para determinação do tamanho médio de cristalito e demais parâmetros cristalográficos. Além disso, a morfologia do pó foi analisada a partir da microscopia eletrônica de varredura (MEV) em microscópio SEM SSX-550 (Shimadzu), acoplado com um espectrômetro de energia dispersiva (EDS), o qual foi utilizado para análise química. As medidas de área superficial específica, diâmetro e volume de poro e isoterma de adsorção do pó foram obtidas por adsorção física de nitrogênio sobre o material, seguindo o modelo proposto por BET (Brunauer, Emmett e Teller). Para a realização deste ensaio, foi utilizado um medidor de área específica Quanta Chrome NOVA-1200E, Surface and Pore Analyser, equipado de software para determinação da área superficial específica. As amostras foram desgaseificadas por $3 \mathrm{~h}$ a $300{ }^{\circ} \mathrm{C}$ para remoção de quaisquer materiais fisissorvido no interior dos poros e na superfície do material.

Atividade catalítica: os testes de oxidação catalítica foram realizados num reator de leito fixo com leito catalítico de $0,1 \mathrm{~g}$ e equipado com controladores de fluxos e sistema

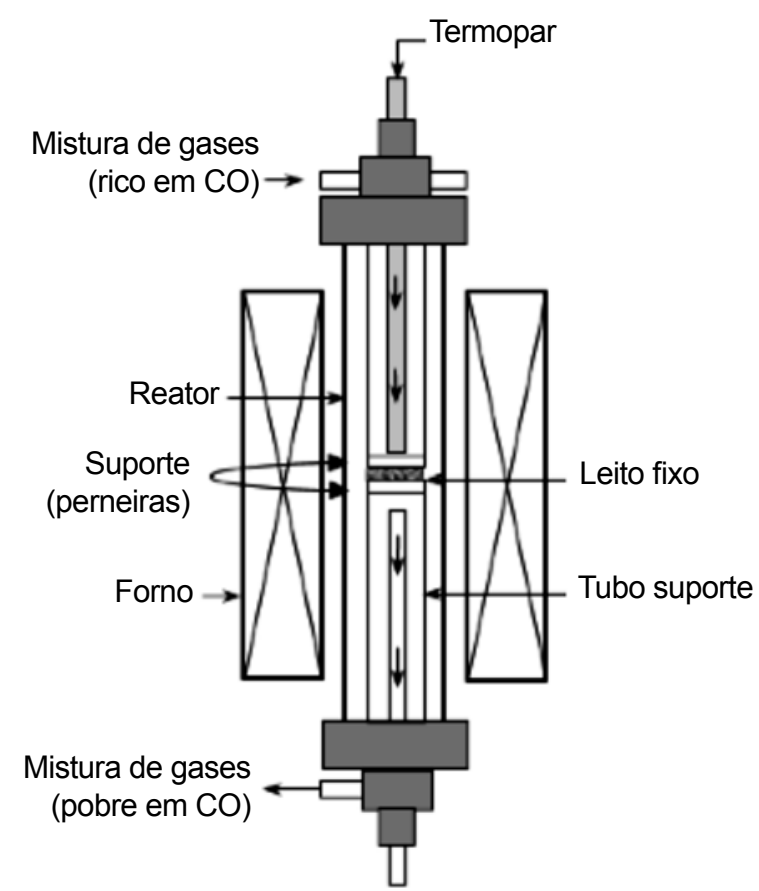

Figura 1: Aparato experimental utilizado nos testes catalíticos.

[Figure 1: Experimental apparatus for catalytic activity experiments.] 
de aquecimento. A Fig. 1 apresenta um esquema do reator de leito fixo usado nos testes. Os gases utilizados nas reações foram oxigênio $\left(\mathrm{O}_{2}\right)$ e monóxido de carbono $(\mathrm{CO})$. Nitrogênio $\left(\mathrm{N}_{2}\right)$ foi utilizado para diluir a mistura até a concentração de trabalho, cerca de $6 \%$. O monóxido de carbono foi fornecido no cilindro em concentração volumétrica de $15 \%$ em hélio (He). A fim de identificar a vazão de operação foram utilizadas vazões de 50 e $100 \mathrm{~mL}$. $\mathrm{min}^{-1}$ com composição fixa e igual a $40 \%$ vol. $(\mathrm{CO}+\mathrm{He}), 15 \%$ vol. $\left(\mathrm{O}_{2}\right)$ e balanço de $\left(\mathrm{N}_{2}\right)$ na faixa de temperatura entre 100 e $550{ }^{\circ} \mathrm{C}$.

\section{RESULTADOS E DISCUSSÃO}

\section{Caracterizações}

A Fig. 2 apresenta os perfis calculado e residual $\mathrm{S}$ (diferença entre o difratograma de raios $\mathrm{X}$ da amostra e do padrão JCPDS $n^{\circ}$ 79001-ICSD) da perovskita $\mathrm{BaCeO}_{3}$. O difratograma apresentou picos característicos da perovskita $\mathrm{BaCeO}_{3}$, mas com a presença de uma fase secundária identificada como carbonato de bário $\left(\mathrm{BaCO}_{3}, \mathrm{JCPDS} \mathrm{n}^{\circ}\right.$ 166091-ICSD), cerca de 3\%, conforme destacada como impureza (\#) na Fig. 2. Segundo a literatura, é comum encontrar a presença de fases secundárias como carbonato de bário e óxido de cério quando a calcinação é realizada em

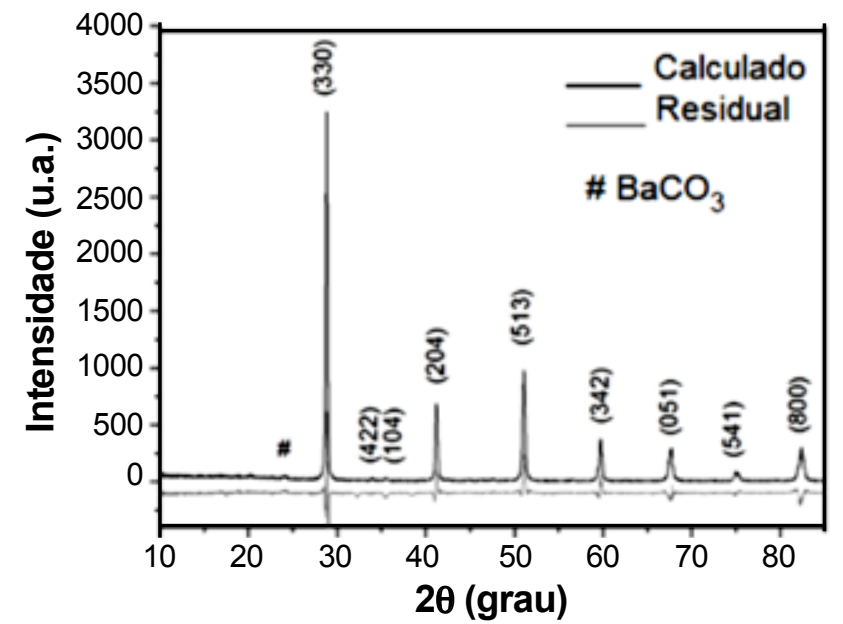

Figura 2: Difratograma de raios $\mathrm{X}$ do pó calcinado a $1000{ }^{\circ} \mathrm{C}$ por $5 \mathrm{~h}$.

[Figure 2: X-ray diffraction pattern of the calcined powder at $1000^{\circ} \mathrm{C}$ for $\left.5 \mathrm{~h}.\right]$ temperatura inferior a $1100{ }^{\circ} \mathrm{C}$ [17]. Através do refinamento de Rietveld foi constatado que o material $\mathrm{BaCeO}_{3}$ apresentou sistema cristalino do tipo ortorrômbico com grupo espacial Pmcn, concordante com a literatura [14, 17-21]. Quando a perovskita $\mathrm{BaCeO}_{3}$ foi sintetizada a partir do método de complexação combinando EDTA-citrato e nas condições experimentais aqui impostas, o tamanho médio de cristalito foi de $133,2 \mathrm{~nm}$, com densidade de $3,38{\mathrm{~g} . \mathrm{cm}^{-3}}^{-3}$ volume de célula unitária igual a $347,89 \mathrm{~nm}^{3}$. Entretanto, dependendo do método de síntese adotado, observam-se variações no tamanho médio de cristalito do $\mathrm{BaCeO}_{3}$, o que pode influenciar diretamente na aplicação do material. A Tabela I resume algumas das condições de síntese empregadas na obtenção do cerato de bário.

A partir da Tabela I observa-se que o tamanho médio de cristalito da perovskita $\mathrm{BaCeO}_{3}$ varia entre 61 e 163 $\mathrm{nm}$ dependendo do método de síntese adotado, como das condições do tratamento térmico para a formação da fase. É notório também que o aumento da temperatura de calcinação favorece a eliminação de fases secundárias, mas com o aumento do tamanho médio de cristalito da perovskita. Apesar dos trabalhos relativos ao cerato de bário, pesquisas não param de avançar, uma vez que as características dos pós não só influenciam fortemente o processamento como também as reações que ocorrem durante a queima, e determinam as propriedades finais do material sintetizado, sendo o conhecimento e o domínio do método a ser empregado fundamental para a síntese dos materiais. Isto demonstra o alto grau de relevância na escolha do método de síntese e das condições de sintetização e calcinação para obtenção de pós cristalinos e monofásicos. A Tabela II apresenta os parâmetros de rede da perovskita $\mathrm{BaCeO}_{3}$ e do carbonato de bário $\left(\mathrm{BaCO}_{3}\right)$ obtidos a partir do difratograma e do refinamento Rietveld. Os parâmetros de rede, volume de célula unitária e densidade das fases demonstram que os resultados obtidos são coerentes com os encontrados na literatura [14, 18-21]. O ajuste do refinamento entre os pontos experimentais e os observados no padrão JCPDS, bem como o valor do parâmetro residual (S) próximo da unidade indica a confiabilidade dos resultados obtidos a partir do refinamento Rietveld.

As Figs. 3 e 4 apresentam as micrografias e isoterma de fisissorção de nitrogênio referente ao cerato de bário obtidas a partir das análises de microscopia eletrônica de varredura (MEV) e método BET, respectivamente. A partir das

Tabela I - Condições experimentais distintas na síntese do $\mathrm{BaCeO}_{3}$. [Table I - Different experimental conditions in the synthesis of $\mathrm{BaCeO}_{3}$.]

\begin{tabular}{cccccc}
\hline Método de síntese & $\begin{array}{c}\text { Temperatura de } \\
\text { calcinação }\left({ }^{\circ} \mathrm{C}\right)\end{array}$ & $\begin{array}{c}\text { Patamar } \\
(\mathrm{min})\end{array}$ & $\begin{array}{c}\text { Tamanho médio de } \\
\text { cristalito }(\mathrm{nm})\end{array}$ & Fase secundária & Referência \\
\hline Pechini & $800-1000$ & 240 & 100 & $\mathrm{BaO}, \mathrm{CeO}_{2}, \mathrm{BaCO}_{3}$ & {$[17]$} \\
Coprecipitação & $950-980$ & $60-300$ & $140-163$ & $\mathrm{BaCO}_{3}$ & {$[20]$} \\
Citrato & $800-900$ & 180 & $61-85$ & $\mathrm{CeO}_{2}, \mathrm{BaCO}_{3}$ & {$[21]$} \\
Complexação combinando & 1000 & 300 & 133 & $\mathrm{BaCO}_{3}$ & $\begin{array}{c}\text { Este } \\
\text { trabalho } \\
\text { EDTA-citrato }\end{array}$ \\
\hline
\end{tabular}


Tabela II - Parâmetros cristalográficos obtidos a partir do refinamento Rietveld. [Table II - Crystallographic parameters obtained from the Rietveld refinement.]

\begin{tabular}{cccc}
\hline Fase & Parâmetros de rede $(\AA)$ & Tamanho de cristalito (nm) & Parâmetro residual, S \\
\hline $\mathrm{BaCeO}_{3}$ & $\mathrm{a}=6,22 ; \mathrm{b}=6,24 ; \mathrm{c}=8,79$ & 133,2 & 1,3 \\
& $\alpha=\beta=\gamma=90^{\circ}$ & & \\
$\mathrm{BaCO}_{3}$ & $\begin{array}{c}\mathrm{a}=5,32 ; \mathrm{b}=8,91 ; \mathrm{c}=6,43 \\
\alpha=\beta=\gamma=90^{\circ}\end{array}$ & 100,0 & \\
\hline
\end{tabular}
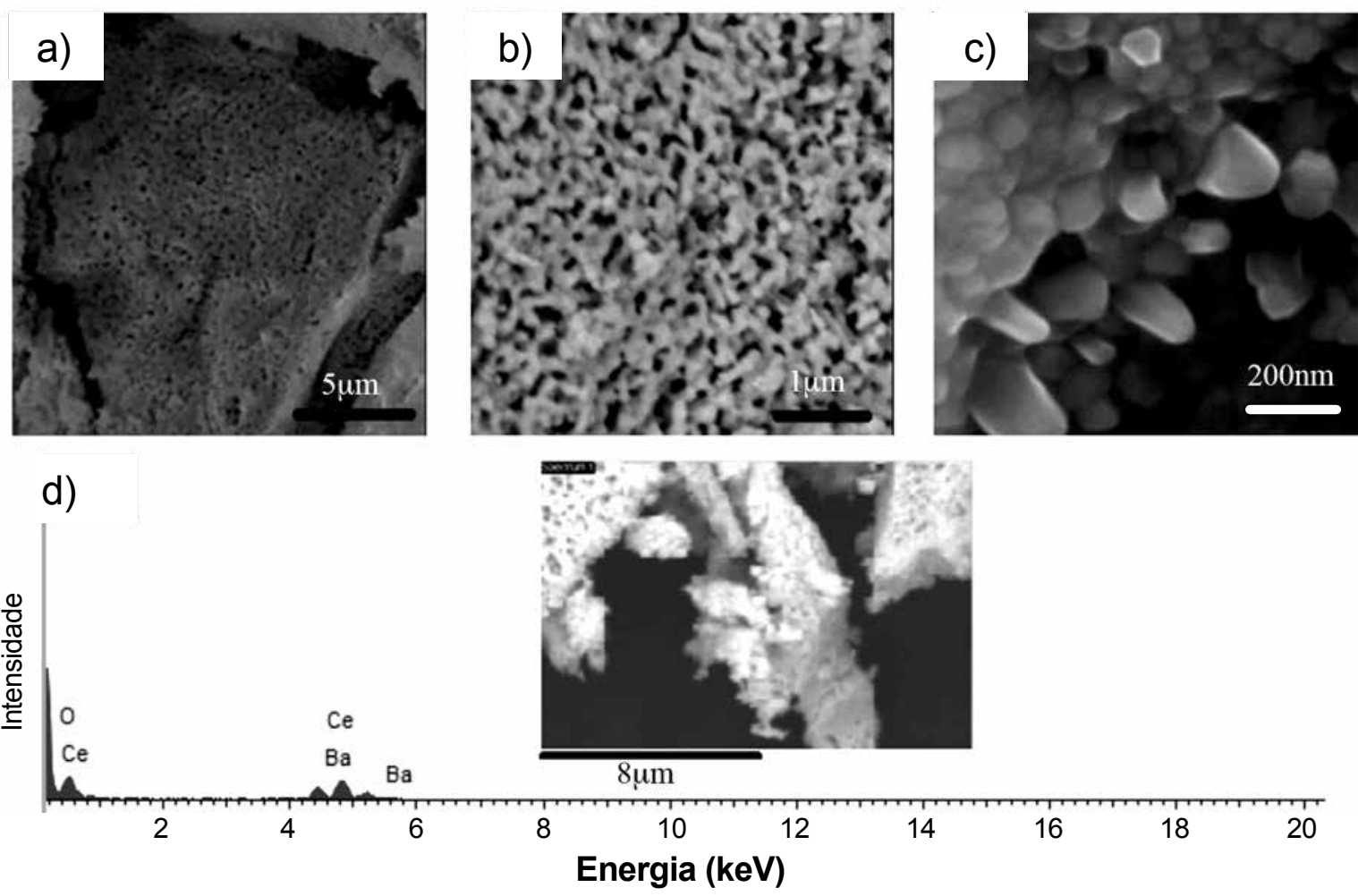

Figura 3: Micrografias obtidas por microscópio eletrônico de varredura (a-c) e espectro de EDS com imagem de $\mathrm{MEV}_{\text {(d) do } \mathrm{BaCeO}}$ calcinado a $1000^{\circ} \mathrm{C}$ por $5 \mathrm{~h}$.

[Figure 3: SEM micrographs (a-c) and EDS spectrum with SEM image (d) of $\mathrm{BaCeO}_{3}$ calcined at $1000^{\circ} \mathrm{C}$ for 5 h.]

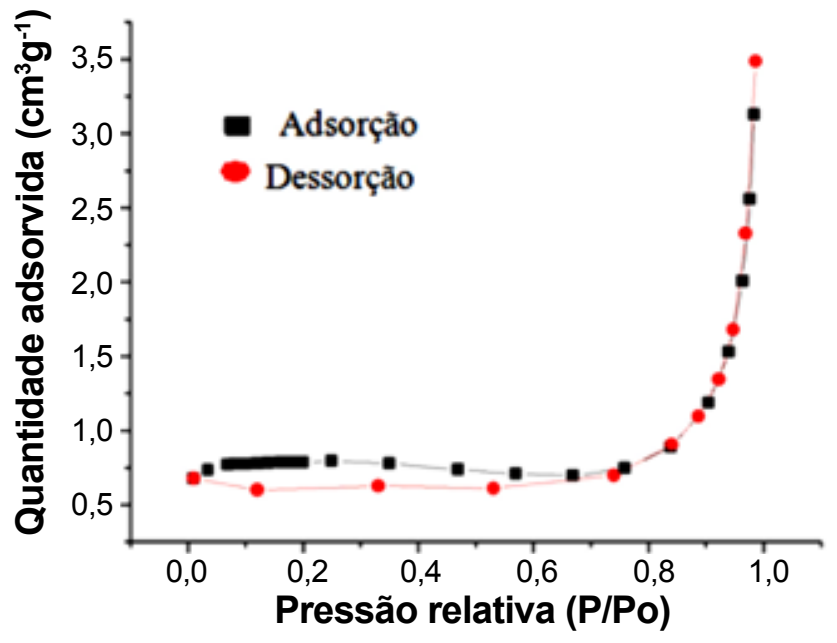

Figura 4: Isoterma de adsorção/dessorção de nitrogênio do $\mathrm{BaCeO}_{3}$. [Figure 4: Nitrogen adsorption/desorption isotherm of the $\mathrm{BaCeO}_{3}$.] micrografias mostradas na Fig. 3 observa-se a presença de um conjunto de aglomerados com formato esféricos irregulares e tamanhos distintos. $\mathrm{Na}$ literatura, a perovskita $\mathrm{BaCeO}_{3}$ apresenta uma distribuição homogênea com tamanho médio de cristalino por volta de $100 \mathrm{~nm}$, o que pode ser atribuído a variações do método de síntese (método Pechini), bem como também do tratamento térmico $\left(980^{\circ} \mathrm{C}\right.$ por $\left.300 \mathrm{~min}\right)$ [17]. Resultado este também concordante com as análises de morfologia (Fig. 3c) e refinamento Rietveld. Pode-se ainda

Tabela III - Análise da composição do cerato de bário. [Table III - Composition analysis of barium cerate.]

\begin{tabular}{ccc}
\hline Elemento & Massa $(\%)$ & Atômico $(\%)$ \\
\hline Bário & 35,42 & 11,14 \\
Cério & 35,42 & 11,01 \\
Oxigênio & 28,84 & 77,85 \\
\hline
\end{tabular}


observar coerência entre o valor esperado da composição química do cerato de bário e o obtido pelo método de síntese adotado, conforme resultados apresentados na Fig. $3 \mathrm{~d}$ e Tabela III.

O cerato de bário apresenta isoterma do tipo II (Fig. 4), encontrado quando a adsorção ocorre em materiais não porosos ou macroporosos [22], resultado este concordante com os apresentados na análise de morfologia do material. São ainda apresentadas as propriedades texturais da perovskita (Tabela IV), como área superficial específica e volume de poro. A área superficial apresentada pelo cerato de bário foi de $2,61 \mathrm{~m}^{2} \cdot \mathrm{g}^{-1}$, dentro da faixa de valores esperada, pois geralmente os valores de área específica dos materiais do tipo perovskita obtidos por métodos convencionais são entre $1 \mathrm{e} 10 \mathrm{~m}^{2} \cdot \mathrm{g}^{-1}$ [23]. Na literatura pode-se observar variações na área superficial para o mesmo material $\left(\mathrm{BaCeO}_{3}\right)$, como por exemplo: 2,1 e $5,8 \mathrm{~m}^{2} \cdot \mathrm{g}^{-1}$ [20] e $6 \mathrm{~m}^{2} \cdot \mathrm{g}^{-1}$ [17]. Tais resultados podem ser justificados pela diferença de temperatura e patamar de calcinação, que pode aumentar ou diminuir o tamanho de grão formado durante o tratamento térmico, assim como também o tipo de método de síntese e suas condições experimentais.

Tabela IV - Propriedades texturais obtidas pelo método BET do $\mathrm{BaCeO}_{3}$.

[Table IV - Textural properties from BET method of $\mathrm{BaCeO}$.]

\begin{tabular}{lc}
\hline Propriedade textural & $\mathrm{BaCeO}_{3}$ \\
\hline Área superficial $\left(\mathrm{m}^{2} \cdot \mathrm{g}^{-1}\right)$ & 2,61 \\
Volume de poro $\left(\mathrm{cm}^{3} \cdot \mathrm{g}^{-1}\right)$ & 0,0048 \\
Diâmetro de poro $(\mathrm{nm})$ & 7,40 \\
\hline
\end{tabular}

\section{Atividade catalítica do $\mathrm{BaCeO}_{3}$}

Visando otimizar as condições para avaliar a atividade catalítica apresentada pelo $\mathrm{BaCeO}_{3}$, fez-se inicialmente um estudo para verificar o efeito da vazão de alimentação da mistura gasosa reacional composta pelo monóxido de carbono, hélio, nitrogênio e oxigênio na conversão total do monóxido de carbono em dióxido de carbono. A Fig. 5 apresenta o efeito das vazões utilizadas $(50$ e 100 mL.min ${ }^{-1}$ ) nos testes catalíticos da perovskita $\mathrm{BaCeO}_{3}$ na faixa de temperatura entre 100 e $450{ }^{\circ} \mathrm{C}$. Conforme Fig. 5 , utilizando a vazão de $50 \mathrm{~mL} \cdot \mathrm{min}^{-1}$ a conversão aumenta com o aumento da temperatura, sendo mais significativa acima de $300^{\circ} \mathrm{C}$. Para a vazão de $100 \mathrm{~mL} \cdot \mathrm{min}^{-1}$ a conversão é constante entre 100 e $300{ }^{\circ} \mathrm{C}$. Observa-se também que na vazão de $50 \mathrm{~mL} \cdot \mathrm{min}^{-1}$ ocorre $50 \%$ de conversão de $\mathrm{CO}$ em $\mathrm{CO}_{2}$ a $335^{\circ} \mathrm{C}\left(\mathrm{T}_{50}\right)$ e uma completa conversão a $400{ }^{\circ} \mathrm{C}$ $\left(\mathrm{T}_{100}\right)$. Já, com a vazão de $100 \mathrm{~mL} \cdot \mathrm{min}^{-1}$ a conversão não atinge $50 \%$ até o limite máximo de temperatura testada (450 ${ }^{\circ} \mathrm{C}$ ), o que provavelmente indica que o tempo de contato entre o gás a ser oxidado e o catalisador não é suficiente com essa vazão de alimentação. A partir deste resultado,

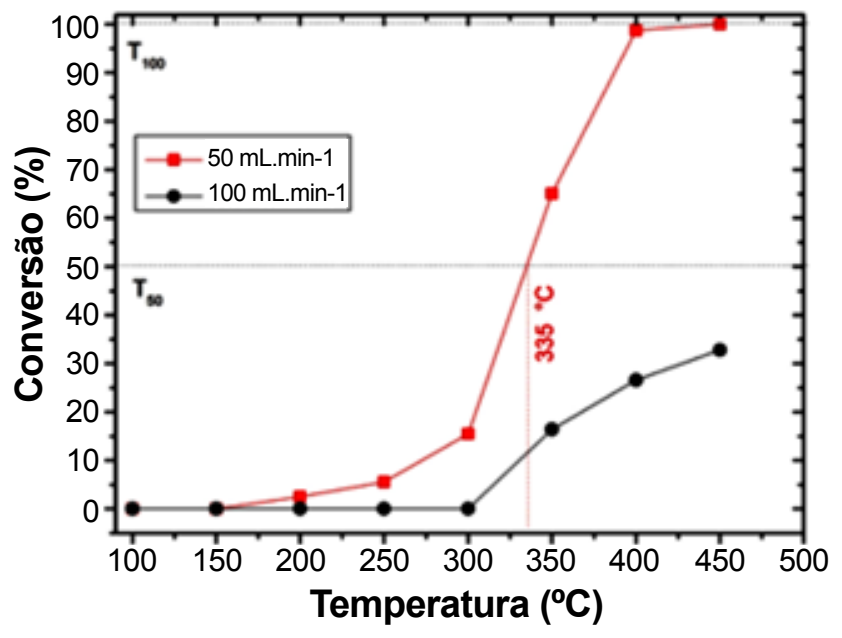

Figura 5: Efeito da vazão de alimentação da mistura reacional utilizada nos testes catalíticos da perovskita $\mathrm{BaCeO}_{3}$.

[Figure 5: Effect of reaction gas flow rate used in catalytic tests of $\mathrm{BaCeO}_{3}$ perovskite.]

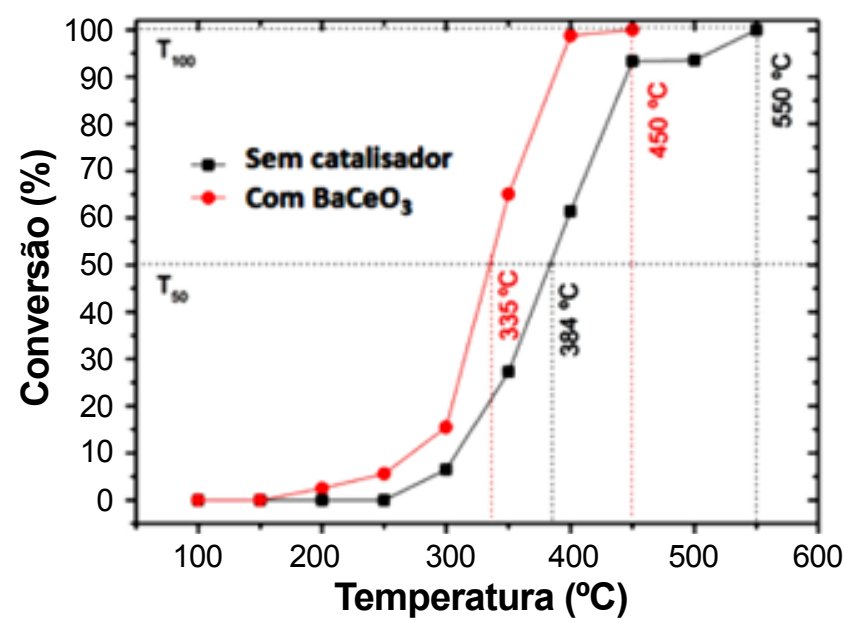

Figura 6: Conversão do monóxido de carbono em função da temperatura com e sem o $\mathrm{BaCeO}_{3}$ como catalisador.

[Figure 6: Conversion of carbon monoxide as a function of temperature with and without $\mathrm{BaCeO}_{3}$ catalyst.]

foram realizados os testes de conversão sem e com a presença do $\mathrm{BaCeO}_{3}$ quando sintetizado pelo método de complexação combinando EDTA-citrato adotando a vazão constante de $50 \mathrm{~mL} \cdot \mathrm{min}^{-1}$ e variando a temperatura de reação entre 100 e $550^{\circ} \mathrm{C}$. A Fig. 6 apresenta a conversão do monóxido de carbono em função da temperatura com e sem a presença do $\mathrm{BaCeO}_{3}$ como catalisador.

Pode-se observar que o $\mathrm{BaCeO}_{3}$ apresenta atividade catalítica na reação de oxidação do $\mathrm{CO}$ na faixa de temperatura verificada $\left(100\right.$ a $\left.550{ }^{\circ} \mathrm{C}\right)$, segundo Fig. 6. O perfil da curva de conversão é similar quando comparada com e sem a presença de catalisador $\left(\mathrm{BaCeO}_{3}\right)$ na reação de oxidação, porém observa-se com o uso do catalisador um aumento na conversão a partir de 200 ${ }^{\circ} \mathrm{C}$, mais significativo a partir de $300{ }^{\circ} \mathrm{C}$, quando ocorre a ativação térmica do catalisador, e com conversão de $50 \%$ a $335{ }^{\circ} \mathrm{C}\left(\mathrm{T}_{50}\right)$ e total praticamente a $400{ }^{\circ} \mathrm{C}\left(\mathrm{T}_{100}\right)$, enquanto sem a presença do $\mathrm{BaCeO}_{3}$ a conversão atingida 
$\mathrm{T}_{50}$ e $\mathrm{T}_{100}$ são 384 e $550{ }^{\circ} \mathrm{C}$, respectivamente. Pode-se concluir que a perovskita apresenta poder catalítico na reação de oxidação do monóxido de carbono, apesar da sua pequena área superficial $\left(2,67 \mathrm{~m}^{2} \cdot \mathrm{g}^{-1}\right)$, classificando essa perovskita como catalisador monolítico para essa reação especificamente. Comparando a atividade catalítica do cerato de bário com a de outras perovskitas binárias testadas na oxidação do monóxido de carbono, podese observar que a perovskita $\mathrm{LaCoO}_{3}$ apresenta uma conversão completa a $200{ }^{\circ} \mathrm{C}$ [10] e em uma temperatura ainda menor $\left(150^{\circ} \mathrm{C}\right) \mathrm{com}$ a substituição do cobalto no sítio B da perovskita por níquel [5]. Tal comportamento pode ser atribuído ao poder catalítico dos metais em relação às terras raras, assim como a maior área superficial

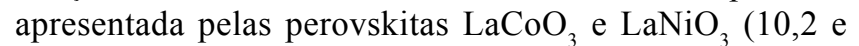
$6,3 \mathrm{~m}^{2} \cdot \mathrm{g}^{-1}$, respectivamente). No entanto, vale salientar que a atividade catalítica das perovskitas depende basicamente da composição química, do grau de cristalinidade e morfologia [23].

\section{CONCLUSÕES}

A partir do difratograma de raios $\mathrm{X}$ e do refinamento pelo método de Rietveld constatou-se que a fase perovskita $\mathrm{BaCeO}_{3}$ foi formada, com cerca de $3 \%$ de impureza $\left(\mathrm{BaCO}_{3}\right)$. $\mathrm{O}$ cerato de bário quando sintetizado pelo método de complexação combinando EDTA-citrato apresenta sistema cristalino ortorrômbico (grupo espacial Pmcn) com tamanho médio de cristalito e área superficial de $133,2 \mathrm{~nm}$ e $2,61 \mathrm{~m}^{2} \cdot \mathrm{g}^{-1}$, respectivamente, e pós com aglomerados contínuos e formato esférico irregular. Com relação à atividade catalítica, na vazão de $50 \mathrm{~mL} \cdot \mathrm{min}^{-1}$ ocorreu maior conversão quando comparado com a vazão de $100 \mathrm{~mL} \cdot \mathrm{min}^{-1}$, sendo mais evidente entre 300 e $400{ }^{\circ} \mathrm{C}$. Além disso, na vazão de $50 \mathrm{~mL}$. $\mathrm{min}^{-1}$ ocorreu conversão total de $\mathrm{CO}$ em $\mathrm{CO}_{2}$ a $400{ }^{\circ} \mathrm{C}$, enquanto que com $100 \mathrm{~mL} \cdot \mathrm{min}^{-1}$ a conversão não atinge $50 \%$ até 450 ${ }^{\circ} \mathrm{C}$. Com o uso da perovskita como catalisador o processo de oxidação é completo a $400{ }^{\circ} \mathrm{C}$, enquanto que na reação sem catalisador a conversão completa é alcançada somente a $550{ }^{\circ} \mathrm{C}$. Dessa forma, apesar da sua pequena área superficial, o cerato de bário é altamente reativo na oxidação do monóxido de carbono.

\section{AGRADECIMENTOS}

Nossos agradecimentos aos laboratórios LMNRC/ PPGEQ/UFRN, NEPGN, LEMOp/UERN e a CAPES (Brasil) pelo suporte financeiro.

\section{REFERÊNCIAS}

[1] P. Lacorre, J.B. Torrance, J. Pannetier, A.I. Nazzal, P.W. Wang, T.C. Huang, J. Solid State Chem. 91 (1991) 225.

[2] W. Kaituo, W. Xuehang, W. Wenwei, L. Yongni, L. Sen, Ceram. Int. 40 (2014) 5997.

[3] G. Valderrama, A. Kiennemann, M.R. Goldwasser, Catal. Today 142 (2008) 133.

[4] S.M. Lima, A.M. Silva, L.O. Costa, J.M. Assaf, G. Jacobs, B.H. Davis, L.V. Mattos, F.B. Noronha, Appl. Catal. A 377 (2010) 181.

[5] K. Rida, M.A. Peña, E. Sastre, A. Martínez-Arias, J. Rare Earth 30 (2012) 210.

[6] H. Wang, J. Liu, Z. Zhao, Y. Wei, C. Xu, Catal. Today 184 (2012) 288.

[7] Y. Wang, T. Su, W. Liu, Q. Chang, G. Qiao, Ceram. Int. 41 (2015) 6863.

[8] D. Medvedev, A. Murashkina, E. Pikalova, A. Demin, A. Podias, P. Tsiakaras, Prog. Mater. Sci. 60 (2014) 72.

[9] A.G. Santos, M. Arab, L. Patout, C. P. Souza, Catalysts 4 (2014) 77.

[10] P. Doggali, S. Kusaba, Y. Teraoka, P. Chankapure, S. Rayalu, N. Labhsetwar, Catal. Commun. 11 (2010) 665.

[11] B. Seyfi, M. Baghalhaa, H. Kazemian, Chem. Eng. J. 148 (2009) 306.

[12] U.G. Singh, J. Li, J.W. Bennett, A.M. Rappe, R. Seshadri, S.L. Scott, J. Catal. 249 (2007) 349.

[13] X. Ouyang, S.L. Scott, J. Catal. 273 (2010) 83.

[14] A.G. Santos, R.R. Silva, A.G.O. Dantas, M.F. Lobato, C.P. Souza, Revista Verde 9 (2014) 149.

[15] A.G. Santos, I.K.F. Costa, F.K.G. Santos E.C.P. Souza, Holos 3 (2015) 30.

[16] R.A. Young, The Rietveld Method, Oxford University Press, New York (1995).

[17] D.W. Lee, J.H. Won, K.B. Shim, Mater. Lett. 57 (2003) 3346.

[18] Y.P. Fu, C.S. Weng, Ceram. Int. 40 (2014) 10793.

[19] X. Chi, J. Zhang, M. Wu, Y. Liu, Z. Wen, Ceram. Int. 39 (2013) 4899.

[20] R. Köferstein, L. Jäger, S.G. Ebbinghaus, J. Mater. Sci. 45 (2010) 6521.

[21] R. Köferstein, D. Hesse, S.G. Ebbinghaus, Solid State Ionics 203 (2011) 52.

[22] R. Ciola, Fundamentos da catálise, S. Paulo, Ed. Moderna, Ed. da Universidade de S. Paulo (1981) 59.

[23] K.K. Sosetsu, The Chemical Society of Japan, Japan Scientific Societies Press, Tokyo, $2^{\text {nd }}$ Ed., 32 (1997) 155.

(Rec. 09/10/2015, Rev. 08/11/2015, 12/01/2016, 23/01/2016, Ac. 21/03/2016) 